\title{
O entendimento de surdos e ouvintes sobre conceitos de Cinemática em um estudo dirigido
}

\section{The understanding of deaf and hearing people about Kinematics concepts in a directed study}

\author{
${ }^{1}$ Klayton Santana Porto klaytonuesb@hotmail.com \\ ${ }^{2}$ Amanda Amantes
}

\section{RESUMO}

O artigo tem como objetivo principal analisar para quais possíveis preditores, em uma classe de surdos e ouvintes, os sujeitos apresentam um desempenho diferenciado na realização de uma atividade de estudo dirigido sobre Cinemática Escalar. O instrumento aplicado averiguou o entendimento explicitado por estudantes surdos e ouvintes na resolução de uma tarefa que requeria deles a interpretação e a compreensão do conteúdo científico mediado pela língua portuguesa. A análise dos dados apresentada se refere a um estudo exploratório, no qual utilizamos testes clássicos para investigar o desempenho dos alunos considerados em termos de escores brutos. Identificamos e averiguamos a influência dos seguintes preditores: série, turma, sexo e audição. Verificamos que houve diferença para os preditores série, turma e audição em relação às séries. Para os demais preditores não houve diferença significativa na explicitação do entendimento do conteúdo abordado por meio da análise realizada.

Palavras chave: Aprendizagem. Ensino de Física. Educação Inclusiva.

\begin{abstract}
The article aims to analyze which possible predictors in a class of deaf and hearing subjects exhibit a different performance in carrying out an activity directed to the study of Kinematics Climbing. The instrument used examined the understanding that was made explicit by deaf and hearing students in solving a task that required them to interpret and understand the scientific content mediated by the Portuguese language. The data analysis presented relates to an exploratory study in which we used classical testing to study the performance of students considered in terms of raw scores tests. We ascertained and identified the influence of the following predictors: number, class, gender and hearing. We observed a difference for the predictors number, class and hearing in relation to the series. For other predictors there was no significant difference in the explicit understanding of content covered by the analysis.
\end{abstract}

Keywords: Learning. Physics Teaching. Inclusive Education.

1 Doutor e Mestre em Ensino, Filosofia e História das Ciências pela Universidade Federal da Bahia. Professor do curso de Lienciatura em Educação do Campo com habilitações em Ciências da Natureza e Matemática no Centro de Ciência e Tecnologia em Energia e Sustentabilidade da UFRB. Universidade Federal do Recôncavo da Bahia - UFRB.

2 Doutora e mestre em Educação pela Universidade Federal de Minas Gerais. Professora do Programa de Pós-graduação em Ensino, Filosofia e História das Ciências da UFBA. Universidade Federal da Bahia - UFBA. 
A inclusão de estudantes com necessidades educacionais especiais nas escolas regulares constitui uma questão importante na educação brasileira. A Política Nacional de Educação Especial, sob o ponto de vista da Educação Inclusiva, delibera a Educação Inclusiva como um modelo de ensino transversal a ser desenvolvido de maneira suplementar ou complementar ao modelo de escolarização de alunos com deficiências, com transtornos globais do desenvolvimento e com altas habilidades, que são matriculados, independente do nível, em classes comuns do ensino regular (BRASIL, 2008 apud MELETTI; BUENO, 2010; FERREIRA; PORTO, 2020).

A Educação Inclusiva deve basear-se no princípio da igualdade, em que respeitar a diferença não é se opor à igualdade, mas sim garantir direitos iguais para atender às necessidades específicas de cada um, considerando que todos são diferentes. Essa ideia é complementada pelo princípio da equidade que, por sua vez, postula o favorecimento de condições diferenciadas para suprir as desigualdades sociais, culturais e econômicas daqueles que se encontram em situação de desvantagem (OLIVEIRA, 2008; RIBEIRO; DUBOC; PORTO, 2020). Dentre as diversas necessidades educacionais especiais, a surdez encontra-se em destaque, pois uma das causas do insucesso da aprendizagem entre as crianças surdas é a dificuldade de desenvolvimento da língua portuguesa como língua materna que, de certa forma, interfere em sua aprendizagem e em seu convívio social (GOLDFELD, 2002; PORTO, 2018).

O interesse pela temática proposta deu-se a partir da constatação da existência dos diversos problemas relacionados à aprendizagem da Física no Ensino Médio por alunos surdos em um ambiente de inclusão. Neste sentido, este trabalho teve como objetivo principal analisar para quais possíveis preditores, em uma classe de surdos e ouvintes, os sujeitos apresentam um desempenho diferenciado na realização de uma atividade de estudo dirigido sobre Cinemática Escalar.

A atividade consistiu em um estudo dirigido que teve o foco na avaliação do entendimento sobre os conceitos, do ponto de vista fenomenológico, mediado pela articulação da língua portuguesa. O atributo "entendimento" foi avaliado a partir da performance específica mobilizada na solução de problemas da Física, e também como capacidade de expor o entendimento sobre os conceitos envolvidos.

O entendimento foi tratado nesta pesquisa dentro de um paradigma cognitivista, em que é explicitado por meio diversos tipos de habilidades, que são utilizadas para compreender os conteúdos, interpretar e analisar os significados e incorporar elementos novos (AMANTES, 2009; PORTO, 2014, 2018). Essas habilidades são interpretadas como estruturas cognitivas que os indivíduos usam em contextos específicos e que variam em complexidade (SCHWARTZ; FISCHER, 2004). Isto significa que uma habilidade mais geral emerge do desenvolvimento de habilidades mais específicas de forma a aumentarem em nível de complexidade.

\section{TEORIA DE HABILIDADES DINÂMICAS}

Para identificar os níveis de complexidade dos diferentes tipos de entendimentos explicitados na realização do estudo dirigido, a perspectiva teórica adotada foi a de níveis hierárquicos estabelecidos a partir da Teoria de Habilidades Dinâmicas (FISCHER, 1980). A Teoria de Habilidades Dinâmicas tenta fornecer ferramentas para a predição de sequências de desenvolvimento e sincronias em qualquer domínio e em qualquer ponto do desenvolvimento, integrando o desenvolvimento comportamental e cognitivo (PORTO, 2018).

O desenvolvimento cognitivo é explicado por uma série de estruturas, consideradas em níveis de complexidade, e por um conjunto de regras de transformação que servem para correlacionar esses níveis uns com os outros. Essas regras de transformação explicitam os passos seguidos por um atributo (no nosso caso, o entendimento) ao passar de forma gradual de um nível para outro (FISCHER, 1980). Os níveis de habilidades podem aumentar gradualmente em termos de complexidade, à medida que uma habilidade específica foi construída, a 
partir de sucessivas reelaborações de habilidades de um nível inferior. Essas habilidades são concebidas como estruturas abstratas de controle que são definidas em termos de ações mentais ou motoras na Teoria de Habilidades Dinâmicas (FISCHER, 1980 apud PORTO, 2014).

Os construtos "habilidades” e "níveis" são relacionados à caracterização original de Piaget (1985) sobre estágios, mas nessa teoria foram refinados em um conjunto de ferramentas que podem ser utilizadas para uma análise mais sistemática de dados observáveis. Dessa forma, ela apresenta um modelo hierárquico que concebe as habilidades como estruturas cognitivas que os alunos usam em contextos específicos, variando em nível de complexidade. Concebe-se uma dimensão multimodal para o desenvolvimento humano e para a evolução do atributo entendimento.

A evolução no entendimento é considerada como um processo em que um novo conceito é compreendido a partir do momento em que o aprendiz consegue interpretar e reinterpretar esse conceito sob diversas perspectivas e situações, as mais distintas possíveis (COELHO, 2011), o que caracteriza o processo de generalização. Assim, a elaboração e reelaboração de significados representa o progresso de um entendimento, cujas habilidades cognitivas servem para generalizar e estender determinado conceito.

Essa teoria nos deu subsídios para sistematizar e elaborar uma taxonomia semelhante à de Parzialle (1998), Dawson (2008), Amantes (2009), Coelho (2011) e Porto (2014; 2018) para que assim se pudesse criar um modelo categórico para a análise de cada uma das questões do estudo dirigido. Esse sistema categórico foi elaborado por meio de um sistema de rubricas, cujas categorias foram obtidas a partir de sucessivas análises das respostas dos alunos para cada item da atividade. As rubricas foram elaboradas levando-se em conta a hierarquia das respostas, obtidas a partir do grau de complexidade do entendimento explicitado, tomando por base a Teoria de Habilidades Dinâmicas (FISCHER, 1980) e a Taxonomia de Complexidade do Entendimento - TCE (AMANTES et al, 2013; PORTO, 2014).

\section{DELINEAMENTO METODOLÓGICO}

Desenvolvemos três atividades de coleta de dados para a pesquisa de mestrado (PORTO, 2014), levando em conta os conceitos básicos de Cinemática Unidimensional. Os instrumentos de coleta de dados elaborados nesta pesquisa foram: um estudo dirigido, uma atividade de construção e interpretação de gráficos e uma atividade envolvendo simulação computacional. Estas três atividades foram elaboradas no intuito de investigar o entendimento dos estudantes acerca do conteúdo abordado, levando-se em consideração diferentes facetas do conhecimento. Entretanto, neste artigo detivemo-nos apenas à análise de uma destas três atividades, o estudo dirigido.

O conteúdo abordado no estudo dirigido foi a Cinemática Unidimensional, tendo sido elencados os seguintes tópicos: (i) conceitos fundamentais (posição, deslocamento, trajetória e referencial); (ii) velocidade e velocidade média; (iii) aceleração e aceleração média; (iv) Movimento Retilíneo Uniforme (MRU) e (v) Movimento Retilíneo Uniformemente Variado (MRUV).

Participaram desta pesquisa 158 estudantes (146 ouvintes e 12 surdos) do Ensino Médio de uma escola pública Estadual do município de Vitória da Conquista - BA. Dessa amostra, 66 alunos eram do primeiro ano (59 ouvintes e 7 surdos), 56 do segundo ano (52 ouvintes e 4 surdos) e 36 do terceiro ano (35 ouvintes e 1 surdo). A coleta foi realizada em um total de 5 turmas, sendo duas de primeiro ano, duas de segundo ano e uma de terceiro ano. A coleta foi realizada utilizando duas aulas da disciplina Física, contando com a participação do professor da disciplina e do intérprete de língua portuguesa para Língua Brasileira de Sinais (LIBRAS) como mediadores na aplicação da atividade. O pesquisador também participou de todas as atividades, a fim de que as possíveis dúvidas dos alunos surdos e ouvintes pudessem ser esclarecidas.

No estudo dirigido foram elencadas questões que permitiram que os estudantes fizessem julgamentos e interpretações de natureza qualitativa e outras que permitiriam que estes fizessem análises quantitativas dos 
fenômenos englobados. A atividade foi respondida individualmente por cada sujeito, para que fosse possível averiguar as respostas de surdos e ouvintes, mediante análise por meio do sistema categórico, elaborado a partir das respostas dos estudantes e da Taxonomia da Complexidade do Entendimento (TCE). A análise procurou avaliar o entendimento dos conceitos científicos, do ponto de vista fenomenológico, apresentados a partir de mediação da língua portuguesa, a fim de verificar se esta vem a ser um fator limitante ou não do entendimento dos alunos, levando-se em consideração os seguintes preditores: série, turma, sexo e audição.

\section{4}

\section{TAXONOMIA DA COMPLEXIDADE DO ENTENDIMENTO}

O sistema categórico elaborado por meio da TCE possibilitou classificar as respostas dos estudantes a partir do nível de complexidade estabelecido do ponto de vista formal. Esse sistema categórico forneceu bases para construirmos a matriz de dados dicotômicos, a partir de 0 e 1, levando-se em consideração uma escala do tipo Guttman (GUTTMAN, 1944). Na escala Guttman, há uma hierarquia presente nas respostas, em que os primeiros itens da escala representam o conhecimento em menor nível e os últimos níveis da escala apresentam os maiores níveis (GUTTMAN, 1944). Essa classificação dos entendimentos na escala Guttman serviu para transformarmos os dados qualitativos, obtidos a partir das respostas dos estudantes no estudo dirigido, em uma escala quantitativa de dados dicotômicos, representativa da TCE.

A matriz de dados dicotômicos criada englobou todas as respostas que os alunos deram a cada um dos itens da atividade, fornecendo-nos o escore bruto. A partir desse escore foram feitos testes estatísticos específicos, de análise clássica, tais como: teste de Levene, Teste ANOVA e teste POS HOC de Bonferroni, para que pudéssemos verificar as diferenças no desempenho dos estudantes em relação a cada um dos preditores elencados inicialmente.

Para verificarmos se os grupos estão variando normalmente realizamos o teste de Levene, a fim de pudéssemos verificar se era possível utilizarmos testes paramétricos para a análise dos dados. Para averiguarmos se a diferença de média para cada um dos preditores foi diferente estatisticamente, fizemos o teste ANOVA. Utilizamos como POS HOC o teste de Bonferroni para, depois de rejeitada a hipótese nula de que as médias são iguais, identificarmos entre quais dos subgrupos da amostra há diferença de média estatisticamente significativa, quando comparados aos pares.

\section{ANÁLISE DOS DADOS}

Encontramos, para a análise com relação às séries, que os grupos estão variando normalmente (L0,95 $(2,155)=0,127 ; \mathrm{p}=0,881)$. Dessa forma, testes paramétricos foram aplicados aosdados da amostra.

Pelo teste ANOVA, $(\mathrm{F} 0,95(2,155)=22,060 ; \mathrm{p}=0,000, \mathrm{p}<0,05)$ verificamos que as médias das séries não são iguais, o que pode ser melhor visualizado no gráfico de escore total para cada série mostrado abaixo: 


\section{Gráfico 1: Escore total para cada série - Estudo Dirigido}

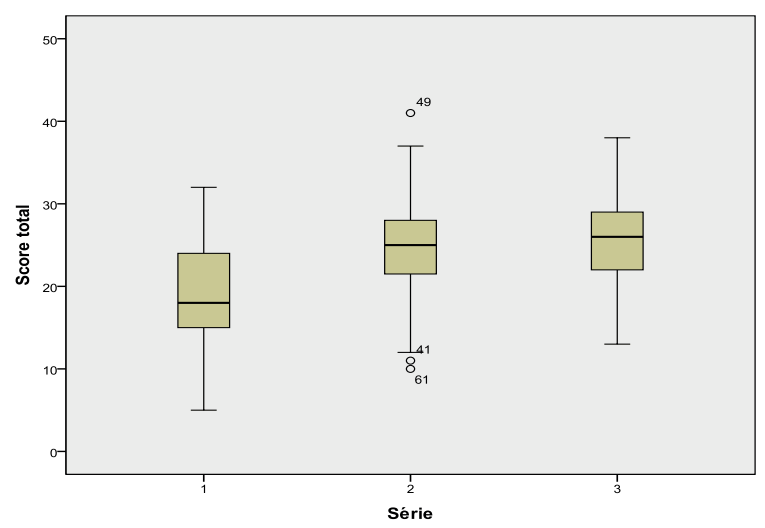

Fonte: dados da pesquisa (2014)

É importante destacar que houve um total de 3 outliers dentre os alunos do $2^{\circ}$ ano, um deste apresentando desempenho acima da média (aluno A49) e dois apresentaram resultados abaixo da média (alunos A41 e A61).

Mesmo que a média tenha se mostrado igual pelo teste ANOVA, pelo gráfico 01 tivemos um indício de que o primeiro ano apresentou um desempenho ligeiramente inferior às demais séries. Por isso, realizamos teste de Bonferroni e o POS HOC, de comparações múltiplas. Os resultados do teste indicaram que houve diferença significativa entre o $1^{\circ}$ e o $2^{\circ}$ ano $(p=0,000 ; p<0,05)$ e entre o $1^{\circ}$ e o $3^{\circ}$ ano $(p=0,000$; $<<0,05)$ e que para $o$ $2^{\circ}$ e $3^{\circ}$ ano não houve uma diferença significativa $(\mathrm{p}=1,000 ; \mathrm{p}>0,05)$, como suspeitado pela análise gráfica. Esse é o primeiro indício de que a maturidade possivelmente influencia o nível de entendimento dos alunos, uma vez que os alunos do $2^{\circ}$ e do $3^{\circ}$ tiveram desempenho semelhante e maior, o que nos leva a concluir que o tempo de acomodar os conceitos é essencial para que seus significados se tornem mais formalizados e, possivelmente, aumentem em nível de complexidade.

Com relação às turmas, verificamos também que, assim como para a análise com relação às séries, os grupos variam normalmente. (L0,95 $(4,153)=0,283, \mathrm{p}=0,889)$; e pelo teste ANOVA, verificamos que as médias das turmas não são iguais $(\mathrm{F} 0,95(4,153)=17,429, \mathrm{p}=0,000, \mathrm{p}<0,05)$. Para sabermos entre quais turmas e as médias foram diferentes, fizemos o teste de Bonferroni e encontramos os seguintes resultados: entre as turmas 1 e $2(\mathrm{p}=0,000) ; 1$ e $3(\mathrm{p}=0,000) ; 1$ e $5(\mathrm{p}=0,000) ; 2$ e $4(\mathrm{p}=0,006) ; 3$ e $4(\mathrm{p}=0,000)$ e 4 e $5(\mathrm{p}=0,000)$, logo a diferença de média entre estas turmas não foi significativa; já entre as turmas 1 e 4 ( $p=0,680)$; 2 e $3(p=1,000)$; 2 e $5(p=1,000)$ e 3 e $5(p=1,000)$ houve uma diferença significativa entre as médias.

Com relação ao sexo, verificamos que os grupos também estão variando normalmente $(\mathrm{L}$ 0,95 $(1,156)=$ $0,72, \mathrm{p}=0,788)$. As médias obtidas foram: 18,10 para o sexo feminino e 16,86 para o sexo masculino. Entretanto, essa diferença não foi significativa estatisticamente $(F 0,95(1,156)=3,470, p=0,064, p>0,05)$.

Para a audição, também verificamos que os grupos estão variando normalmente $(\operatorname{L0,95}(1,156)=0,386$, $\mathrm{p}=0,535)$. Verificamos que não houve diferença significativa para entre as médias dos surdos e dos ouvintes $(\mathrm{F} 0,95(1,156)=1,121 \mathrm{e} \mathrm{p}=0,729, \mathrm{p}>0,05)$, conforme podemos ver no gráfico 02, que representa o escore total de acordo a audição: 


\section{Gráfico 2: Escore total de acordo a audição - Estudo Dirigido}

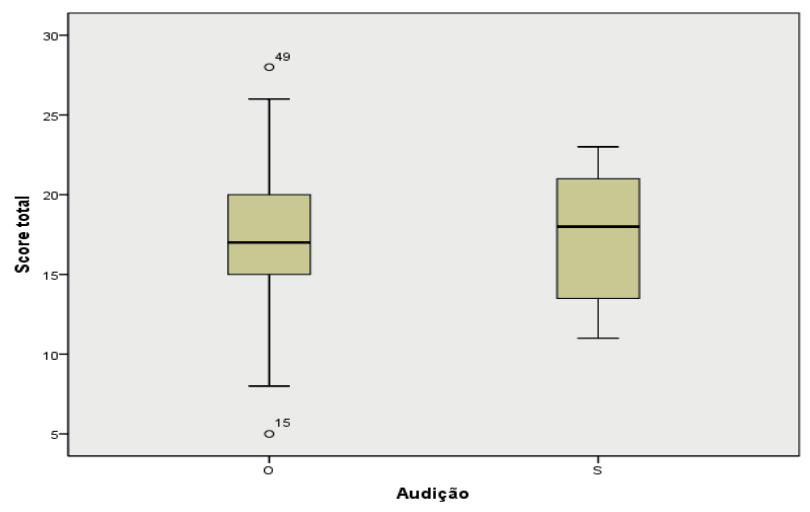

Fonte: dados da pesquisa (2014)

Entretanto, quando analisamos o preditor audição separadamente em relação à cada turma, percebemos que há diferença nos desempenhos de surdos e ouvintes. Essa diferença é significativa entre os surdos e ouvintes para as três séries, conforme podemos ver no gráfico 03, que representa o escore total de acordo a audição e série:

\section{Gráfico 3: Escore total de acordo a audição e série - Estudo Dirigido}

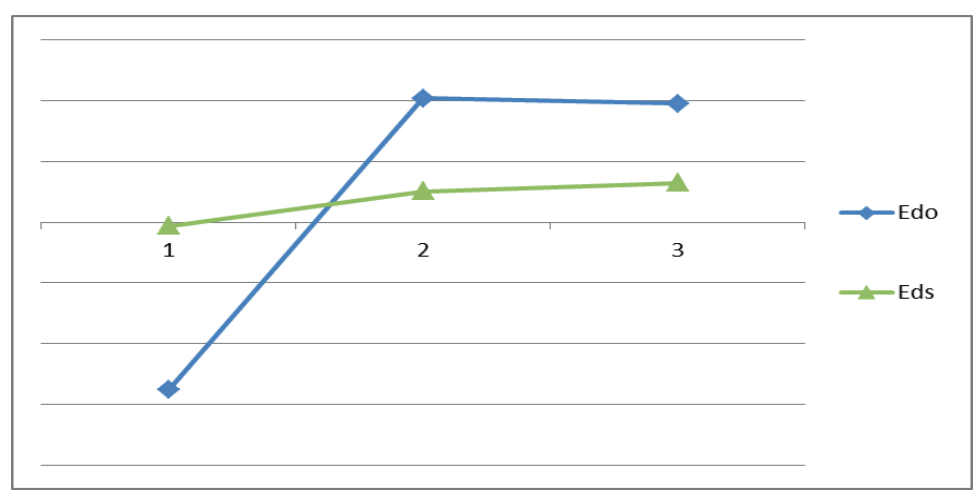

Fonte: dados da pesquisa (2014)

No gráfico 03, Edo representa o escore para o estudo dirigido dos estudantes ouvintes e Eds representa o escore para os surdos.

Essa diferença pode ser verificada quando analisamos o gráfico 03, que nos mostra que os surdos do primeiro ano apresentaram um desempenho melhor que os ouvintes para essa série; entretanto, para o segundo e terceiro anos os ouvintes apresentaram um desempenho superior aos surdos. Essa diferença de resultado pode ser em virtude do pouco número de surdos em relação aos ouvintes, que nos limita a identificar efeitos mais gerais na amostra como um todo.

De maneira geral, podemos perceber que houve diferença significativa das médias, a partir dos preditores analisados, somente em relação às séries e em relação a algumas turmas, pois houve uma diferença significativa do $1^{\circ}$ ano para o $2^{\circ}$ ano e do $1^{\circ}$ ano para o $3^{\circ}$ ano, não havendo, entretanto, diferença significativa entre o $2^{\circ}$ e $o$ $3^{\circ}$ ano. Para os demais preditores (sexo e audição) não houve diferença significativa, considerando-se os escores dos dados do estudo dirigido, quando analisados isoladamente. Entretanto, verificamos diferenças entre surdos e ouvintes quando levamos em conta a análise de desempenho para cada uma das séries separadamente. 
De um modo geral, os alunos do terceiro ano apresentaram um desempenho melhor que as demais séries, isso nos dá evidência de que, mesmo que um conteúdo não seja revisto é necessário um tempo de amadurecimento para que um conteúdo seja compreendido em maior nível de complexidade. Como o primeiro ano teve um desempenho menor que o segundo e que o terceiro, podemos dizer que esse desempenho está associado à memória de curto prazo, pois estes tinham visto a matéria recentemente: eles não tiveram tempo suficiente para acomodar o conhecimento de forma que atingissem níveis maiores de complexidade. Os alunos do segundo e do terceiro ano, embora não tenham visto o conteúdo recentemente, já apresentam um amadurecimento em outros conteúdos da Física que podem ajudar na acomodação desse conteúdo, uma vez que estes estudaram outros conceitos que, de forma subjacente, podem ajudar no entendimento da Cinemática. Isso nos mostra que, mesmo que um conteúdo não seja revisitado, é necessário que haja um tempo de amadurecimento para que o entendimento se torne mais bem articulado, uma vez que não se aprende tudo na forma mais complexa na primeira instrução.

\section{CONSIDERAÇÕES FINAIS}

O entendimento é um atributo que evolui no tempo, e este caráter temporal está associado às recursivas interações entre o sujeito e o objeto de estudo. Deste modo, um novo conhecimento é construído através de sucessivas elaborações e reestruturações de um quadro previamente existente, subtendendo à mobilização de diferentes habilidades para relacionar conteúdos, interpretar significados e incorporar novos elementos a este panorama prévio.

Neste estudo, identificamos que houve diferença de desempenho somente em relação às séries e às turmas. A análise mostrou que houve diferença entre as médias do primeiro e do segundo ano e entre o primeiro e o terceiro ano. Não houve diferença significativa entre o segundo e o terceiro ano. Esse pode ser um primeiro indício de que a maturidade possivelmente influencia, uma vez que os alunos do $2^{\circ}$ e do $3^{\circ}$ ano tiveram um tempo maior para acomodar os conceitos. Esse resultado mostra a importância da memória a longo prazo.

O estudo dirigido serviu para que os estudantes pudessem retomar o conteúdo após o mesmo ter sido acomodado na mente, uma vez que os alunos tinham sido apresentados ao conteúdo algum tempo atrás. O $1^{\circ}$ ano viu o conteúdo recentemente, com isso os alunos não tiveram tempo suficiente para amadurecer e acomodar os conceitos, do ponto de vista de teoria.

Embora na amostra geral essa diferença não seja significativa, quando olhamos para cada série em particular percebemos diferenças entre os estudantes surdos e ouvintes. Isso nos dá indícios de que a língua portuguesa vem a ser um fator limitante para os surdos em uma atividade conceitual na forma de estudo dirigido em que predomina o uso da língua portuguesa escrita.

Do ponto de vista educacional esses resultados podem levar os educadores a compreenderem a importância da adoção de novas estratégias de ensino por meio da utilização de instrumentos didáticos, tais como as atividades utilizadas em nossa pesquisa, que façam com que não haja diferença na aprendizagem entre os surdos e os ouvintes em um contexto de inclusão.

Pretendemos, desse modo, dar continuidade na análise, realizando outras análises em termos de proficiência por meio de modelamento Rasch, a fim de que possamos melhor explicar as possíveis diferenças entre os preditores que elencamos neste artigo, levando em conta os dados obtidos com o estudo dirigido, a atividade de construção e interpretação de gráficos e com a atividade da simulação computacional. Buscaremos, como continuidade de pesquisa, verificar se há ou não diferença no entendimento de surdos e ouvintes para cada uma das três atividades em termos de proficiência. Referências 


\section{REFERÊNCIAS}

AMANTES, A. Contextualização no Ensino de Física: Efeitos sobre a evolução do entendimento dos estudantes. Tese de Doutorado, UFMG, 2009, 275p.

AMANTES, A; MAIA, E; MARINHO, R; COELHO, G; FERNANDES, F. An analytical tool to evoluate conceptual understanding, ESERA, 2013.

COELHO, G. R. A evolução do entendimento dos estudantes em eletricidade: Um estudo longitudinal. Tese de Doutorado, UFMG, 2011, 173p.

DAWSON, T.L. The Lectical' ${ }^{\text {TM }}$ Assessment System, 2008. Disponível em http://www.lectica.info. Acesso em: nov.2016.

FERREIRA, P. N. P.; PORTO, K. S. Práticas Pedagógicas desenvolvidas com alunos com deficiência: um estudo de caso em uma escola do campo de Feira de Santana - BA. Revista Brasileira de Educação do Campo, v. 5, p. e7913, 3 jul. 2020.

FISCHER, K. W. A theory of cognitive development: the control and construction of hierarchies of skills. Psychological Review, v. 87, 1980. 477-531.

GOLDFELD, M. A. A criança surda: linguagem e cognição. São Paulo: Plexus, 2009.

GUTTMAN, L. A basis for scaling qualitative data. American Sociological Review, n. 9, 1944, p. 139-150.

MELETTI, S. M. F.; BUENO, J. G. S. A escolarização de alunos com deficiência: uma análise dos indicadores sociais no Brasil (1997-2006). In: Reunião Anual da ANPED, 33.; 2010, Caxambú. Educação no Brasil: o balanço de uma década, 2010. p.1-17.

OLIVEIRA, E. da S. G. de, Distúrbios de conduta. In. DELOU, C. M. C. et al. Fundamentos teóricos e metodológicos da Inclusão. Curitiba: IESDE, 2008.

PARZIALE, J.; FISCHER, K. W. The practical use of skill theory in classrooms. In: STERNBERG, R. J.; WILLIAMS, W. M. (Ed.). Intelligence, instruction and assessment. 1998, p. 96-110.

PIAGET, J. A linguagem e o pensamento da criança. São Paulo: Martins Fontes, 1985.

PORTO, K. S. Avaliando o entendimento de estudantes surdos e ouvintes de ensino médio sobre Cinemática em um contexto de Educação Inclusiva. Dissertação de Mestrado, UFBA/UEFS, 2014, 143p.

PORTO, K. S. A argumentação e o entendimento de estudantes surdos e ouvintes sobre Cinemática. Tese de Doutorado, UFBA/UEFS, 2018, 267p.

RIBEIRO, S. L.; DUBOC, M. J. O.; PORTO, K. S. Docência no Ensino Superior em tempos de inclusão educacional: um convite à reflexão. Revista Educação, Cultura e Sociedade, v. 10, n. 2, 2020. 